\title{
On some nondecaying potentials and related Jost solutions for the heat conduction equation *
}

\author{
B. Prinari ${ }^{\dagger}$ \\ Dipartimento di Fisica dell'Università and Sezione INFN \\ I-73100 Lecce \\ ITALY
}

November 20, 2018

\begin{abstract}
Potentials of the heat conduction operator constructed by means of 2 binary Bäcklund transformations are studied in detail. Corresponding Darboux transformations of the Jost solutions are introduced. We show that these solutions obey modified integral equations and present their analyticity properties.
\end{abstract}

\section{Introduction}

In this article we investigate into the direct and inverse scattering transform for the heat conduction operator

$$
L=-\partial_{x_{2}}+\partial_{x_{1}}^{2}-u\left(x_{1}, x_{2}\right)
$$

in the case in which $u$ is a real function with "ray" type behavior. More exactly, $u$ is supposed to be rapidly decaying in all directions on the $x$-plane with the exception of some finite number of directions, where it has finite and nontrivial limits, i.e.

$$
u_{n, \pm}\left(x_{1}\right)=\lim _{x_{2} \rightarrow \pm \infty} u\left(x_{1}-2 \mu_{n} x_{2}, x_{2}\right), \quad n=1,2, \ldots, N
$$

for $N$ real constants $\mu_{n}$. The spectral theory of operator $L$ with potential in this class is interesting per se and because it is associated to the Kadomtsev-Petviashvili equation in its version called KPII [1]-[3]

$$
\left(u_{t}-6 u u_{x_{1}}+u_{x_{1} x_{1} x_{1}}\right)_{x_{1}}=-3 u_{x_{2} x_{2}} .
$$

In investigating KPII equation it was found [ 1 that there are significant differences with respect to the case of KPI (equation (1.3) with opposite sign in the rhs). Specifically, the inverse problem cannot be formulated as a Riemann-Hilbert boundary value problem [5]-[7]. This results from the fact that for the associated spectral problem there exist eigenfunctions which, though bounded, are nowhere analytic in the spectral parameter. The role of the Riemann-Hilbert problem is now played by what is usually called a $\bar{\partial}-$ problem. The basic idea is to compute the $\bar{\partial}$-derivative of these eigenfunctions (modified

*Work supported in part by PRIN 97 "Sintesi"

$\dagger$ e-mail: prinari@le.infn.it 
Jost solutions) and then to exploit the Cauchy-Green formula to get a linear Fredholm integral equation for such solutions (under the assumption that the homogeneous integral equation has no nontrivial solutions). One can prove the smoothness of the potential $u(x, t)$ and also some other properties [8]. Anyway, it is always assumed that the potential is rapidly decaying for $x_{1}^{2}+x_{2}^{2} \rightarrow \infty$ (which excludes in particular the presence of line solitons) and a rigorous investigation of the IST and of the properties of solutions of KPII equation with nontrivial (for instance, one-dimensional) asymptotic behavior is still missing. Such extension of the spectral theory for the heat operator would provide the possibility to extend correspondingly the class of solutions of the KPII equation.

The same problem has been faced for the nonstationary Schrödinger equation in the framework of the so-called resolvent approach [9]- -13 . This extension in the general case resulted to be particularly involved, in particular as far as the definition of spectral data and their characterization equations are concerned. Some general results were obtained in [12] with some (partially implicit) assumptions. Even though it was not possible to define precisely the subclass of potentials satisfing these assumptions, it was shown that potentials belonging to this special subclass can be obtained via Bäcklund transformations (BT's). For getting some experience with a case less involved from a mathematical point of view but sufficiently general, it was considered the case of potentials obtained by applying recursively binary BT's to an arbitrary decaying potential and describing $N$ solitons superimposed to a generic background [14]. At least in the case $N=1$ it was possible to obtain explicitly the resolvent, the Jost solutions and the spectral data and to show that they satisfy all the required assumptions [13]. Moreover, there is evidence that the structural properties of the spectral data can be even more complicated in the general case.

Spectral theory of the operator (1.1) with ray potential is essentially more involved than the standard case of rapidly decaying potential. One of the problems is that the standard integral equations defining the Jost solution and its dual become senseless, since the Green's function is slowly decaying at space infinity and cannot ensure convergency. In Sec. 2, after reviewing some properties of Green's function and Jost solutions for the heat conduction equation in the case of rapidly decaying potentials, we propose an extension of these integral equations which allows also to include potentials with ray type behavior and which in the one-dimensional limit give the standard integral equations for the Jost solutions of $\mathrm{KdV}$ equation. We call the solutions of these modified integral equations Jost-like solutions. In Sec. 3 we consider the potentials obtained by superimposing, via binary Bäcklund transformations, one soliton to a generic decaying background. A significant difference with respect to the case of the nonstationary Schrödinger operator is due to the fact that in order to get a real potential we are obliged to use as functions generating the BT's the Jost solution and dual Jost solution computed for purely imaginary values of the spectral parameter and thus an accurate study of their properties at these values is required. We get the explicit expression of the potential and we are able to formulate the conditions to be imposed on the parameters of the Bäcklund transformation in order to obtain a real and regular solution. Moreover, we obtain also the explicit expression of the Jost-like solutions and study their spectral properties. Finally, in Sec. 4 we iterate this procedure to get two solitons on a generic background. Also in this case we determine the regularity conditions for the solutions obtained by these means and we explicitly compute Jost solutions and study their spectral properties. An interesting feature of these solutions describing two solitons on a background is that, unlike KPI case, the directions of the solitons are not fixed by the parameters of the Bäcklund transformations but depend also on the "constants" of integration of the Darboux procedure. 
If the matrix which appears in the expression of the potential is diagonal, the two BT's simply "superimpose" the two solitons with only a phase shift. But in the general case, that is when both off-diagonal entries of such matrix are different from zero, the solitons are rotated with respect to the directions determined by the single BT's. When one of the off-diagonal entries is null but the other one is not, only one soliton is rotated while the other one is unchanged and we could say that the "interaction" does not preserve the soliton direction. If a row or a column in matrix $C_{2}$ is made up of zeros the resulting potential has three "rays". Note that all these features are absent in the case of KPI equation. In fact, for KPI the soliton directions do not depend on the choice of such matrix [14. Another difference with respect to the case of KPI equation is that the KPI wave soliton emerging from the background at large distances divides the $x$-plane into two regions with different asymptotic behavior. In the first region (to the left of the soliton) the original potential $u(x)$ is modified at large distances by an exponentially decreasing term, while in the second region (to the right) by a term decreasing as $1 / x_{2}^{3}$. In the case of KPII the corrections are always decaying at infinity faster that any power of $x_{2}$.

\section{On Jost solutions}

In the standard case one introduces the complex spectral parameter $k=k_{\Re}+i k_{\Im}$ and defines the Jost solution $\Phi(x, k)$ and dual Jost solution $\Psi(x, k)$ as the solutions of the heat conduction equation and its dual, respectively, such that

$$
\begin{aligned}
& \chi(x, k)=e^{i k x_{1}+k^{2} x_{2}} \Phi(x, k) \\
& \xi(x, k)=e^{-i k x_{1}-k^{2} x_{2}} \Psi(x, k)
\end{aligned}
$$

satisfy the integral equations

$$
\begin{aligned}
& \chi(x, k)=1+\int d x^{\prime} G_{0}\left(x-x^{\prime}, k\right) u\left(x^{\prime}\right) \chi\left(x^{\prime}, k\right) \\
& \xi(x, k)=1+\int d x^{\prime} G_{0}\left(x^{\prime}-x, k\right) u\left(x^{\prime}\right) \xi\left(x^{\prime}, k\right)
\end{aligned}
$$

where the Green's function $G_{0}(x, k)$ is given by

$$
G_{0}(x, k)=-\frac{\operatorname{sign} x_{2}}{2 \pi} \int d \alpha \theta\left(\alpha\left(\alpha+2 k_{\Re}\right) x_{2}\right) e^{-i \alpha x_{1}-\alpha(\alpha+2 k) x_{2}} .
$$

It is evident from expression (2.8) that $G_{0}$ and, consequently, $\chi$ and $\xi$ are nowhere analytic in the complex $k$-plane. Functions $\chi$ and $\xi$ are bounded for all $x_{1}, x_{2}$ (see for instance 15]) and

$$
\lim _{k \rightarrow \infty} \chi(x, k)=\lim _{k \rightarrow \infty} \xi(x, k)=1 .
$$

The Jost solutions defined by (2.4) and (2.5) obey the following normalization and completness conditions

$$
\begin{aligned}
& \int d x_{1} \Psi\left(x_{1}, x_{2}, k+p\right) \Phi\left(x_{1}, x_{2}, k\right)=2 \pi \delta(p) \\
& \int d k_{\Re} \Psi\left(x_{1}^{\prime}, x_{2}, k\right) \Phi\left(x_{1}, x_{2}, k\right)=2 \pi \delta\left(x_{1}-x_{1}^{\prime}\right)
\end{aligned}
$$


One can check that

$$
G_{0}(x, k)=\frac{\operatorname{sign} k_{\Re}}{2 i \pi\left|x_{1}\right|}\left[e^{2 i k_{\Re}\left(x_{1}+2 k_{\Im} x_{2}\right)}-1\right]+o\left(\left|x_{1}\right|^{-1}\right)
$$

and

$$
G_{0}(x, k)=-\frac{\operatorname{sign} k_{\Re}}{4 \pi k x_{2}}+o\left(\left|x_{2}\right|^{-1}\right) .
$$

Despite these asymptotics are discountinuous at $k_{\Re}=0$, the Green's function is not and one can easily check from (2.8) that

$$
G_{0}\left(x, i k_{\Im}\right)=-\frac{\theta\left(x_{2}\right)}{2 \sqrt{\pi x_{2}}} e^{-\frac{\left(x_{1}+2 k_{\Im} x_{2}\right)^{2}}{4 x_{2}}}
$$

which is real and, up to the exponential factor $e^{-k_{\Im} x_{1}-k_{\Im}^{2} x_{2}}$, is the well-known GaussWeierstrass kernel (or heat kernel). Then $\chi$ and $\xi$ can be computed at purely imaginary values of the spectral parameter $k$ and they are real as well.

Solvability of the integral equations (2.6)-(2.7) under some small norm assumptions was proved in [16] and thanks to (2.11) it is easy to show that $\chi(x, k)$ and $\xi(x, k)$ have the asymptotic behavior

$$
\begin{aligned}
\lim _{\left|x_{1}\right| \rightarrow \infty} \chi(x, k) & =1 \\
\lim _{\left|x_{1}\right| \rightarrow \infty} \xi(x, k) & =1
\end{aligned}
$$

Moreover, one can prove the following propositions.

Proposition 1 Let $u$ be a real, regular and rapidly decaying potential. The modified Jost solutions $\chi$ and $\xi$ obey for any $n \in \mathbb{N}$ the following asymptotics

$$
\begin{aligned}
\lim _{x_{2} \rightarrow \pm \infty} x_{2}^{-n}\left(\chi\left(x, i k_{\Im}\right)-1\right) & =0, \\
\lim _{x_{2} \rightarrow \pm \infty} x_{2}^{-n}\left(\xi\left(x, i k_{\Im}\right)-1\right) & =0 .
\end{aligned}
$$

Proof. Let us consider the integral equation (2.6) for $\chi$. We have for $k_{\Im}=\kappa \in \mathbb{R}$

$$
\chi(x, i \kappa)-1=-\frac{1}{2 \sqrt{\pi}} \int d x^{\prime} \frac{\theta\left(x_{2}-x_{2}^{\prime}\right)}{\sqrt{x_{2}-x_{2}^{\prime}}} e^{-\frac{\left(x_{1}-x_{1}^{\prime}+2 \kappa\left(x_{2}-x_{2}^{\prime}\right)\right)^{2}}{4\left(x_{2}-x_{2}^{\prime}\right)}} u\left(x^{\prime}\right) \chi\left(x^{\prime}, i \kappa\right)
$$

and we can make shift and proper change of variables to get

$$
\chi(x, i \kappa)-1=-\frac{1}{\sqrt{\pi}} \int d x^{\prime} \theta\left(x_{2}^{\prime}\right) e^{-\left(x_{1}^{\prime}\right)^{2}}(u \chi)\left(x_{1}-2 x_{1}^{\prime}\left|x_{2}^{\prime}\right|^{1 / 2}+2 \kappa\left|x_{2}^{\prime}\right|, x_{2}-\left|x_{2}^{\prime}\right|, i \kappa\right) .
$$

Note that

$$
\begin{aligned}
& \left|\int d x_{1}^{\prime} \int_{0}^{+\infty} d x_{2}^{\prime} e^{-\left(x_{1}^{\prime}\right)^{2}}(u \chi)\left(x_{1}-2 x_{1}^{\prime}\left|x_{2}^{\prime}\right|^{1 / 2}+2 \kappa\left|x_{2}^{\prime}\right|, x_{2}-\left|x_{2}^{\prime}\right|, i \kappa\right)\right| \leq \\
\leq & \int_{-\infty}^{+\infty} d x^{\prime} e^{-\left(x_{1}^{\prime}\right)^{2}}\left|(u \chi)\left(x_{1}-2 x_{1}^{\prime}\left|x_{2}^{\prime}\right|^{1 / 2}+2 \kappa\left|x_{2}^{\prime}\right|, x_{2}-\left|x_{2}^{\prime}\right|, i \kappa\right)\right| .
\end{aligned}
$$

Since $\chi$ is bounded and $u$ is in the Schwartz class the limit $\left|x_{2}\right| \rightarrow \infty$ can be exchanged with the integral and the integrand decays as fast as $u$, that is faster than any power of $x_{2}$. The same result holds for $\xi$. 
Proposition 2 Let $u$ be a real potential in the Schwartz class. The modified Jost solutions $\chi$ and $\xi$ for the heat conduction equation with potential $u$ are such that $\chi\left(x, i k_{\Im}\right) \neq$ $0, \xi\left(x, i k_{\Im}\right) \neq 0 \forall x \in \mathbb{R}^{2}$, or equivalently, taking into account asymptotics (2.10)-(2.10),

$$
\begin{aligned}
& \chi\left(x, i k_{\Im}\right)>0 \\
& \xi\left(x, i k_{\Im}\right)>0,
\end{aligned} \quad \forall x \in \mathbb{R}^{2} .
$$

Proof. In order to simplify the notation in the following we will put $k_{\Im}=\kappa \in \mathbb{R}$. Let us write the solution of the integral equation (2.6) as the (formal) Neumann series

$$
\chi(x, i \kappa)=\sum_{n=0}^{\infty} \chi_{n}(x, i \kappa)
$$

where

$$
\begin{aligned}
& \chi_{0}(x, i \kappa)=1 \\
& \chi_{n}(x, i \kappa)=-\frac{1}{2 \sqrt{\pi}} \int d x^{\prime} \frac{\theta\left(x_{2}-x_{2}^{\prime}\right)}{\sqrt{x_{2}-x_{2}^{\prime}}} e^{-\frac{\left[\left(x_{1}-x_{1}^{\prime}\right)+2 \kappa\left(x_{2}-x_{2}^{\prime}\right)\right]^{2}}{4\left(x_{2}-x_{2}^{\prime}\right)}} u\left(x^{\prime}\right) \chi_{n-1}\left(x^{\prime}, i \kappa\right)
\end{aligned}
$$

Obviously we have to consider the class of potentials for which the existence of solution of the integral equation, that is the convergency of the Neumann series, is estabilished (see [16] and the following remark).

Now let us assume that for all $x \in \mathbb{R}^{2}$

$$
|u(x)|<U\left(x_{2}\right)
$$

with $U$ such that

$$
\int_{0}^{+\infty} d x_{2}^{\prime}\left|U\left(x_{2}-x_{2}^{\prime}\right)\right|<1 .
$$

If we introduce

$$
a(x)=\int d x^{\prime} \frac{\theta\left(x_{2}-x_{2}^{\prime}\right)}{\sqrt{x_{2}-x_{2}^{\prime}}} e^{-\frac{\left[\left(x_{1}-x_{1}^{\prime}\right)+2 \kappa\left(x_{2}-x_{2}^{\prime}\right)\right]^{2}}{4\left(x_{2}-x_{2}^{\prime}\right)}}\left|u\left(x^{\prime}\right)\right|,
$$

due to (2)-2.21) we have

$$
0<\frac{a(x)}{2 \sqrt{\pi}}<\frac{1}{2} .
$$

Then by induction on $n$ one can easily prove that

$$
\left|\chi_{n+1}(x, i \kappa)\right|<\left(\frac{1}{2}\right)^{n+1}
$$

so that

$$
|\chi(x, i \kappa)-1|<1 .
$$

The main problem when dealing with a potential $u(x)$ not vanishing in all directions at large distances is that the integral equations (2.6) and (2.7) cannot be applied 
as the Green's function is slowly decaying at space infinity. We suggest the following modification of these integral equations:

$$
\begin{aligned}
\chi(x, k) & =1+\int_{-k_{\Im} \infty}^{x_{1}} d y_{1} \int d x^{\prime} \partial_{y_{1}} G_{0}\left(y_{1}-x_{1}^{\prime}, x_{2}-x_{2}^{\prime}, k\right) u\left(x^{\prime}\right) \chi\left(x^{\prime}, k\right), \\
\xi(x, k) & =1+\int_{k \Im \infty}^{x_{1}} d y_{1} \int d x^{\prime} \partial_{y_{1}} G_{0}\left(x_{1}^{\prime}-y_{1}, x_{2}^{\prime}-x_{2}, k\right) u\left(x^{\prime}\right) \xi\left(x^{\prime}, k\right),
\end{aligned}
$$

where the order of operations is explicitly prescribed. Here and below we use notations of the type $k_{\Im} \infty$ in the limits of integrals to indicate the sign of infinity. If the solution of this equation exists and is bounded, then like in the standard case

$$
\begin{aligned}
\lim _{x_{1} \rightarrow-k_{\Im} \infty} \chi(x, k) & =1, \\
\lim _{x_{1} \rightarrow k_{\Im} \infty} \xi(x, k) & =1, \quad k_{\Im} \neq 0,
\end{aligned}
$$

while in contrast to (2.13) and (2.14) they can be different from 1 in the opposite direction. These modified integral equations are applicable to the simplest case of a potential of type (1.2), i.e. to the case $u(x)=u\left(x_{1}\right)$ and one recovers the standard one-dimensional integral equation for Jost solution of KdV equation.

Since the general case is extremely involved, we are studying here the special but rather wide subclass of potentials of type (1.2) that is obtained by applying recursively the so-called binary Bäcklund transformations [17] with complex spectral parameter to a decaying potential. As we are interested in the spectral properties of potentials $u$ having nontrivial limits, following an approach close to the one in [19], we study also the corresponding Darboux transformations furnishing the Jost solutions of the transformed potentials and their analytical properties, as well as transformations of the spectral data.

\section{Binary Bäcklund transformations}

We have at our disposal in 17 and [18] a rather simple and transparent method for performing binary Bäcklund transformations of the potential $u$ and corresponding Darboux transformations of solutions of (1.1) and its dual. Let $u_{0}$ be a smooth, real and rapidly decaying potential and $L_{0}$ the corresponding heat operator

$$
L_{0}=-\partial_{x_{2}}+\partial_{x_{1}}^{2}-u_{0}(x) .
$$

A new potential $u_{0}^{\prime}(x)$ can be generated through an elementary BT

$$
L_{0}^{\prime} B_{0}=B_{0} L_{0}
$$

using a gauge operator

$$
B_{0}=\partial_{x_{1}}-\left(\partial_{x_{1}} \log \varphi_{0}(x)\right) .
$$

If we impose (3.28) we find that

$$
u_{0}^{\prime}(x)=u_{0}(x)-2 \partial_{x_{1}}^{2} \log \varphi_{0}(x)
$$

and that $\varphi_{0}$ has to be a solution of the original spectral problem, that is

$$
\left(-\partial_{x_{2}}+\partial_{x_{1}}^{2}-u_{0}(x)\right) \varphi_{0}(x)=0 .
$$


Let us consider also the spectral operator dual to (3.27), that is

$$
L_{0}^{d}=\partial_{x_{2}}+\partial_{x_{1}^{2}}-u_{0}(x)
$$

and the gauge operator

$$
B_{0}^{d}=\partial_{x_{1}}+\left(\partial_{x_{1}} \log \varphi_{0}(x)\right) .
$$

Eq. (3.28) is equivalent to the corresponding dual relation

$$
B_{0}^{d} L_{0}^{\prime d}=L_{0}^{d} B_{0}^{d} .
$$

Moreover, given any two solutions of the spectral problem (3.27) and of its dual (3.32), say $\phi_{0}$ and $\psi_{0}$, their Darboux transforms $\phi_{0}^{\prime}$ and $\psi_{0}^{\prime}$, defined by

$$
\begin{gathered}
\phi_{0}^{\prime}=B_{0} \phi_{0} \\
B_{0}^{d} \psi_{0}^{\prime}=\psi_{0}
\end{gathered}
$$

solve, respectively, the spectral problem for the transformed potential and its dual up to annihilators of $B_{0}^{d}$, that is $L_{0}^{\prime} \phi_{0}^{\prime}=0$ and $B_{0}^{d} L_{0}^{\prime} \psi_{0}^{\prime}=0$.

One can easily check that if $f$ and $g$ solve (3.31) and its dual, respectively, then

$$
\partial_{x_{2}}(f(x) g(x))=-\partial_{x_{1}} W(f(x), g(x))
$$

where $W$ is standard Wronskian of $f$ and $g$, that is

$$
W(f(x), g(x))=f(x) \partial_{x_{1}} g(x)-g(x) \partial_{x_{1}} f(x) .
$$

Now let us consider an inverse elementary BT

$$
L_{0}^{\prime} B_{0}^{\prime}=B_{0}^{\prime} L_{1}
$$

through the gauge

$$
B_{0}^{\prime}=\partial_{x_{1}}+\left(\partial_{x_{1}} \log \psi_{0}^{\prime}(x)\right) .
$$

In this case we have

$$
u_{1}(x)=u_{0}^{\prime}(x)-2 \partial_{x_{1}}^{2} \log \psi_{0}^{\prime}
$$

where $\psi_{0}^{\prime}$ is an arbitrary solution of

$$
\left(\partial_{x_{2}}+\partial_{x_{1}}^{2}-u_{0}^{\prime}(x)\right) \psi_{0}^{\prime}(x)=0 .
$$

Now we perform direct and inverse elementary Bäcklund transformations (3.28) and (3.34) and study the properties of operator $L_{1}$, omitting all intermediate constructions associated to operator $L_{0}^{\prime}$. First of all, from (3.30) and (3.41) we see that

$$
u_{1}(x)=u_{0}(x)-2 \partial_{x_{1}}^{2} \log \Delta_{1}(x)
$$

where we introduced

$$
\Delta_{1}(x)=\varphi_{0}(x) \psi_{0}^{\prime}(x) .
$$

Moreover, using (3.30), from (3.31) and (3.42) we find that $\varphi_{0}$ and $\psi_{0}^{\prime}$ have to be chosen in such a way that they satisfy the following system of equations

$$
\left\{\begin{array}{l}
\left(-\partial_{x_{2}}+\partial_{x_{1}}^{2}-u_{0}(x)\right) \varphi_{0}(x)=0 \\
\left(\partial_{x_{2}}+\partial_{x_{1}}^{2}-2\left(\partial_{x_{1}} \log \varphi_{0}(x)\right) \partial_{x_{1}}\right) \Delta_{1}(x)=0
\end{array} .\right.
$$


It is clear that a sufficient condition for $u_{1}$ in (3.43) to be real is to choose $\Delta_{1}$ real, but this means that $\varphi_{0}$ cannot be an arbitrary solution of the original spectral problem. In particular, one can choose a real solution such as the Jost solution computed for purely imaginary value of spectral parameter, that is

$$
\varphi_{0}(x)=\Phi_{0}\left(x, i \kappa_{1}\right)
$$

In order to obtain $\psi_{0}^{\prime}(x)$, and consequently $\Delta_{1}$, we consider the Darboux transform (3.36) of a solution $\psi_{0}(x)$ of the spectral problem $L_{0}^{d} \psi_{0}=0$ and we choose

$$
\psi_{0}(x)=\Psi_{0}\left(x, i \alpha_{1}\right)
$$

where $\Psi_{0}$ is the Jost solution of the spectral problem (3.32) computed for $k=i \alpha_{1}$, $\alpha_{1} \in \mathbb{R}$. With this choice we get

$$
\psi_{0}^{\prime}(x)=\frac{1}{\varphi_{0}(x)}\left[C\left(x_{2}\right)+\int_{-\left(\kappa_{1}-\alpha_{1}\right) \infty}^{x_{1}} d x_{1}^{\prime} \Psi_{0}\left(x_{1}^{\prime}, x_{2}, i \alpha_{1}\right) \Phi_{0}\left(x_{1}^{\prime}, x_{2}, i \kappa_{1}\right)\right]
$$

and the integral is convergent due to the asymptotic behaviors (2.13) and (2.14). "Constant" of integration $C$ depends, in general, on $x_{2}$ as well, but one can check that $\psi_{0}^{\prime}$ is indeed a solution of (3.42) iff $C$ does not depend on $x_{2}$. So finally we get

$$
\Delta_{1}(x)=c_{1}+\int_{-\left(\kappa_{1}-\alpha_{1}\right) \infty}^{x_{1}} d x_{1}^{\prime} \Phi_{0}\left(x_{1}^{\prime}, x_{2}, i \kappa_{1}\right) \Psi_{0}\left(x_{1}^{\prime}, x_{2}, i \alpha_{1}\right),
$$

where $c_{1}$ and $\alpha_{1}, \kappa_{1}$ are real constants with $\alpha_{1} \neq \kappa_{1}$.

Taking into account (3.49), it is always possible to formulate a small norm condition which ensures regularity of the dressed potential (3.43). Indeed, Prop. 2 proves that $\Phi_{0}\left(x, i k_{\Im}\right)$ and $\Psi_{0}\left(x, i k_{\Im}\right)$ are real and strictly positive for all $x \in \mathbb{R}^{2}$; then the integral in (3.49) is a monotonic function of $x_{1}$ and if we choose $c_{1}$ such that

$$
\left(\kappa_{1}-\alpha_{1}\right) c_{1} \geq 0,
$$

eq. (3.49) gives a function $\Delta_{1}$ which is regular, has no zeros in the $x$-plane and has the same sign as $\kappa_{1}-\alpha_{1}$. Consequently, condition (3.50) ensures regularity of potential (3.43). Finally, one can easily show that $\Delta_{1}$ indeed superimposes one soliton on the generic (smooth and rapidly decaying) background $u_{0}$ along the direction $x_{1}+\left(\kappa_{1}+\alpha_{1}\right) x_{2}=$ const.

\subsection{Darboux procedure}

We need now to express the Jost solutions $\Phi_{1}$ and $\Psi_{1}$ of the spectral equations

$$
L_{1} \Phi_{1}=0, \quad L_{1}^{d} \Psi_{1}=0
$$

in terms of the Jost solutions $\Phi_{0}$ and $\Psi_{0}$ of the original spectral problem, that is we have to construct the Darboux version of the binary BT. From (3.39) we see that if $\phi_{0}^{\prime}$ satisfies $L_{0}^{\prime} \phi_{0}^{\prime}=0$ and we define $\phi_{1}$ through

$$
B_{0}^{\prime} \phi_{1}=\phi_{0}^{\prime}
$$

then $\phi_{1}$ is such that $B_{0}^{\prime} L_{1} \phi_{1}=0$. So, taking into account definition of $B_{0}^{\prime}$ in (3.40), from (3.52) we get

$$
\partial_{x_{1}}\left(\phi_{1}(x, k) \psi_{0}^{\prime}(x)\right)=\phi_{0}^{\prime}(x, k) \psi_{0}^{\prime}(x) .
$$


In order to have transformations parametrized by constants and not by functions of $x_{2}$ obeying some differential equation, it would be convenient to integrate this equation from infinity to $x_{1}$. From the other side, it is natural to use (3.35) to get $\phi_{0}^{\prime}$, that is

$$
\phi_{0}^{\prime}(x, k)=\partial_{x_{1}} \phi_{0}(x, k)-\frac{\partial_{x_{1}} \Phi_{0}\left(x, i \kappa_{1}\right)}{\Phi_{0}\left(x, i \kappa_{1}\right)} \phi_{0}(x, k),
$$

and in particular to choose $\phi_{0}(x, k) \equiv \Phi_{0}(x, k)$. However, in this case $\phi_{0}^{\prime}$ has at large $x_{1}$ the same behavior as $\Phi_{0}$, and then from (3.48) it follows that for $\left(k_{\Im}-\alpha_{1}\right)\left(k_{\Im}-\kappa_{1}\right)<0$ it is not possible to integrate (3.53) on the infinite interval. Then we integrate (3.53) from some $x_{1}^{0}$ to $x_{1}$ getting

$$
\phi_{1}(x, k)=\frac{1}{\psi_{0}^{\prime}(x)}\left[C_{1}^{\prime}\left(x_{2}, k\right)+\int_{x_{1}^{0}}^{x_{1}} d x_{1}^{\prime} \phi_{0}^{\prime}\left(x_{1}^{\prime}, x_{2}, k\right) \psi_{0}^{\prime}\left(x_{1}^{\prime}, x_{2}\right)\right] .
$$

$C_{1}^{\prime}\left(x_{2}, k\right)$ is a "constant" of integration and $\phi_{1}(x, k)$ is a solution of $L_{1} \phi_{1}=0$ iff

$$
\partial_{x_{2}}\left(C_{1}^{\prime}\left(x_{2}, k\right)\right)=-\left.W\left(\phi_{0}^{\prime}(x, k), \psi_{0}^{\prime}(x)\right)\right|_{x_{1}=x_{1}^{0}} .
$$

Substituting $\phi_{0}^{\prime}$ given by (3.54) into (3.55) and integrating by parts we get

$$
\phi_{1}(x, k)=\Phi_{0}(x, k)+\frac{1}{\psi_{0}^{\prime}(x)}\left[C_{1}\left(x_{2}, k\right)-\int_{-\left(k_{\Im}-\alpha_{1}\right) \infty}^{x_{1}} d x_{1}^{\prime} \Phi_{0}\left(x_{1}^{\prime}, x_{2}, k\right) \Psi_{0}\left(x_{1}^{\prime}, x_{2}, i \alpha_{1}\right)\right],
$$

where

$C_{1}\left(x_{2}, k\right)=C_{1}^{\prime}\left(x_{2}, k\right)-\Phi_{0}\left(x_{1}^{0}, x_{2}, k\right) \psi_{0}^{\prime}\left(x_{1}^{0}, x_{2}\right)+\int_{-\left(k_{\Im}-\alpha_{1}\right) \infty}^{x_{1}^{0}} d x_{1}^{\prime} \Phi_{0}\left(x_{1}^{\prime}, x_{2}, k\right) \Psi_{0}\left(x_{1}^{\prime}, x_{2}, i \alpha_{1}\right)$.

One can check that due to (3.1) and (3.57) $C_{1}$ does not depend on $x_{2}$. Finally, we obtain from (3.55) a solution of the heat conduction equation with potential $u_{1}$ parametrized by an arbitrary function of $k$

$$
\phi_{1}(x, k)=\Phi_{0}(x, k)-\frac{\Phi_{0}\left(x, i \kappa_{1}\right)}{\Delta_{1}(x)}\left[C_{1}(k)+\int_{-\left(k_{\Im}-\alpha_{1}\right) \infty}^{x_{1}} d x_{1}^{\prime} \Phi_{0}\left(x_{1}^{\prime}, x_{2}, k\right) \Psi_{0}\left(x_{1}^{\prime}, x_{2}, i \alpha_{1}\right)\right]
$$

and we can introduce also the solution $F_{1}$ defined by

$$
F_{1}(x, k)=\Phi_{0}(x, k)-\frac{\Phi_{0}\left(x, i \kappa_{1}\right)}{\Delta_{1}(x)} \int_{-\left(k_{\Im}-\alpha_{1}\right) \infty}^{x_{1}} d x_{1}^{\prime} \Phi_{0}\left(x_{1}^{\prime}, x_{2}, k\right) \Psi_{0}\left(x_{1}^{\prime}, x_{2}, i \alpha_{1}\right) .
$$

Eq. (3.59) shows that $F_{1}$ has the same analytical properties of $\Phi_{0}$ and an additional pole for $k=i \alpha_{1}$. In fact, since $\Phi_{0}$ is continuous for $k=i \alpha_{1}$, it follows from (2.9) that

$$
F_{1}\left(x, k_{\Re}+i\left(\alpha_{1}+0\right)\right)-F_{1}\left(x, k_{\Re}+i\left(\alpha_{1}-0\right)\right)=-2 \pi \frac{\Phi_{0}\left(x, i \kappa_{1}\right)}{\Delta_{1}(x)} \delta\left(k_{\Re}\right)
$$

and then

$$
F_{1}(x, k)=\frac{1}{i k+\alpha_{1}} \frac{\Phi_{0}\left(x, i \kappa_{1}\right)}{\Delta_{1}(x)}+\mathrm{reg}
$$


where reg denotes terms which are regular in the limit $k \rightarrow i \alpha_{1}$. Moreover one can check that there exist limits

$$
\lim _{x_{1} \rightarrow \pm \infty} e^{i k x_{1}+k^{2} x_{2}} F_{1}(x, k)=A_{1}( \pm, k)
$$

where

$$
A_{1}( \pm, k)=1+\frac{\left(\kappa_{1}-\alpha_{1}\right)}{\left(i k+\alpha_{1}\right)} \theta\left( \pm\left(\kappa_{1}-\alpha_{1}\right)\right)
$$

and so the solution of the modified integral equation (2.23) with potential $u_{1}$ is given by

$$
\chi_{1}(x, k)=e^{i k x_{1}+k^{2} x_{2}} \Phi_{1}(x, k)
$$

with

$$
\Phi_{1}(x, k)=\frac{F_{1}(x, k)}{A_{1}\left(-\operatorname{sign} k_{\Im}, k\right)} .
$$

Indeed, since $\Phi_{1}$ satisfies the heat conduction equation with potential $u_{1}$ we have

$$
\begin{aligned}
& \int d x^{\prime}\left(\partial_{x_{1}} G_{0}\left(x-x^{\prime}, k\right)\right) u_{1}\left(x^{\prime}\right) \chi_{1}\left(x^{\prime}, k\right)=\int d x^{\prime}\left(\partial_{x_{1}} G_{0}\left(x-x^{\prime}, k\right)\right) \times \\
& \times\left(-\partial_{x_{2}^{\prime}}+\partial_{x_{1}^{\prime}}^{2}-2 i k \partial_{x_{1}^{\prime}}\right) \chi_{1}\left(x^{\prime}, k\right)
\end{aligned}
$$

and $\partial_{x_{1}}$ cancels all slowly decaying terms of the Green's function (see (2.11) and (2.12) so we can integrate by parts in the right hand side getting

$$
\int d x^{\prime} \partial_{x_{1}} G_{0}\left(x-x^{\prime}, k\right) u_{1}\left(x^{\prime}\right) \chi_{1}\left(x^{\prime}, k\right)=\partial_{x_{1}} \chi_{1}(x, k) .
$$

It follows that

$$
\begin{aligned}
& 1+\int_{-k_{\Im} \infty}^{x_{1}} d y_{1} \int d x^{\prime} \partial_{y_{1}} G_{0}\left(y_{1}-x_{1}^{\prime}, x_{2}-x_{2}^{\prime}, k\right) u_{1}\left(x^{\prime}\right) \chi_{1}\left(x^{\prime}, k\right)= \\
= & 1+\int_{-k_{\Im} \infty}^{x_{1}} d y_{1} \partial_{y_{1}} \chi_{1}\left(y_{1}, x_{2}, k\right)=1+\chi_{1}(x, k)-\lim _{x_{1} \rightarrow-k_{\Im} \infty} \chi_{1}(x, k)
\end{aligned}
$$

and this completes the proof since, due to definition (3.63), $\lim _{x_{1} \rightarrow-k_{\Im} \infty} \chi_{1}(x, k)=1$. Note that from (3.62) and (3.64) and taking into account (3.1), we see that if $\kappa_{1} \alpha_{1}<0$ $\Phi_{1}$ has no poles in the complex $k$-plane; if $\kappa_{1} \alpha_{1}>0$ and $0<\alpha_{1}<\kappa_{1}$ or $\kappa_{1}<\alpha_{1}<0$ it has a pole at $k=i \alpha_{1}$ while if $\alpha_{1}<\kappa_{1}<0$ or $0<\kappa_{1}<\alpha_{1}$ it has a pole for $k=i \kappa_{1}$ (in any case the pole corresponds to the one which is smaller by modulo). So, strictly speaking, $\Phi_{1}$ cannot be considered a Jost solution since it may have a pole. We will call it Jost-like solution.

As far as the dual solutions are concerned, in the same way as before we get

$$
\begin{gathered}
\psi_{1}(x, k)=\Psi_{0}(x, k)-\frac{\Psi_{0}\left(x, i \alpha_{1}\right)}{\Delta_{1}(x)}\left[D_{1}(k)+\int_{\left(k_{\Im}-\kappa_{1}\right) \infty}^{x_{1}} d x_{1}^{\prime} \Psi_{0}\left(x_{1}^{\prime}, x_{2}, k\right) \Phi_{0}\left(x_{1}^{\prime}, x_{2}, i \kappa_{1}\right)\right] \\
\Upsilon_{1}(x, k)=\Psi_{0}(x, k)-\frac{\Psi_{0}\left(x, i \alpha_{1}\right)}{\Delta_{1}(x)} \int_{\left(k_{\Im}-\kappa_{1}\right) \infty}^{x_{1}} d x_{1}^{\prime} \Psi_{0}\left(x_{1}^{\prime}, x_{2}, k\right) \Phi_{0}\left(x_{1}^{\prime}, x_{2}, i \kappa_{1}\right) .
\end{gathered}
$$


Note that $\Upsilon_{1}$, as a function of the spectral parameter $k$, has the same analytical properties of $\Psi_{0}$ and an additional pole for $k=i \kappa_{1}$. Indeed, using (2.9) one can easily check from (3.66) that

$$
\Upsilon_{1}(x, k)=\frac{1}{i k+\kappa_{1}} \frac{\Psi_{0}\left(x, i \alpha_{1}\right)}{\Delta_{1}(x)}+\mathrm{reg} .
$$

If we compute its asymptotic behavior, we see that

$$
\lim _{x_{1} \rightarrow \pm \infty} e^{-i k x_{1}-k^{2} x_{2}} \Upsilon_{1}(x, k)=B_{1}( \pm, k)
$$

with

$$
B_{1}( \pm, k)=1+\frac{\left(\alpha_{1}-\kappa_{1}\right)}{\left(i k+\kappa_{1}\right)} \theta\left( \pm\left(\kappa_{1}-\alpha_{1}\right)\right)
$$

and then, to obtain the dual Jost solution, satisfying the modified integral equation (2.24), we have to consider

$$
\Psi_{1}(x, k)=\frac{\Upsilon_{1}(x, k)}{B_{1}\left(\operatorname{sign} k_{\Im}, k\right)} .
$$

Using (3.69) and (3.67) we can prove that $\Psi_{1}$ has no poles iff $\alpha_{1} \kappa_{1}<0$. For $0<\alpha_{1}<\kappa_{1}$ or $\kappa_{1}<\alpha_{1}<0$ it has a pole at $k=i \alpha_{1}$ while if $\alpha_{1}<\kappa_{1}<0$ or $0<\kappa_{1}<\alpha_{1}$ it has a pole behavior at $k=i \kappa_{1}$.

\section{Two solitons on a generic background}

Now we are going to iterate once the procedure illustrated above. We consider

$$
\phi_{1}(x, k)=\phi_{0}(x, k)-a_{1}(x)\left[C_{1}(k)+\int_{-\left(k_{\Im}-\alpha_{1}\right) \infty}^{x_{1}} d x_{1}^{\prime} \phi_{0}\left(x_{1}^{\prime}, x_{2}, k\right) \psi_{0}\left(x_{1}^{\prime}, x_{2}, i \alpha_{1}\right)\right]
$$

and

$$
\psi_{1}(x, k)=\psi_{0}(x, k)-b_{1}(x)\left[D_{1}(k)+\int_{\left(k_{\Im}-\kappa_{1}\right) \infty}^{x_{1}} d x_{1}^{\prime} \psi_{0}\left(x_{1}^{\prime}, x_{2}, k\right) \phi_{0}\left(x_{1}^{\prime}, x_{2}, i \kappa_{1}\right)\right]
$$

where $\phi_{0}$ and $\psi_{0}$ are, respectively, the Jost solution of the original spectral problem and of its dual, that is

$$
\phi_{0}(x, k)=\Phi_{0}(x, k), \quad \psi_{0}(x, k)=\Psi_{0}(x, k),
$$

and we introduced

$$
\begin{aligned}
& a_{1}(x)=\frac{\phi_{0}\left(x, i \kappa_{1}\right)}{\Delta_{1}(x)} \quad b_{1}(x)=\frac{\psi_{0}\left(x, i \alpha_{1}\right)}{\Delta_{1}(x)} \\
& \Delta_{1}(x)=c_{1}+\int_{-\left(\kappa_{1}-\alpha_{1}\right) \infty} d x_{1}^{\prime} \phi_{0}\left(x_{1}^{\prime}, x_{2}, i \kappa_{1}\right) \psi_{0}\left(x_{1}^{\prime}, x_{2}, i \alpha_{1}\right)
\end{aligned}
$$

and $C_{1}(k)$ and $D_{1}(k)$ are arbitrary functions of $k$ such that $C_{1}\left(i k_{\Im}\right), D_{1}\left(i k_{\Im}\right)$ are real, so that $\phi_{1}\left(x, i k_{\Im}\right)$ and $\psi_{1}\left(x, i k_{\Im}\right)$ are real and can be used to generate the binary BT.

We proved that $a_{1}(x)$ and $b_{1}(x)$ are solutions of the spectral problem (3.27) with potential $u_{1}$ and of its dual, respectively. Now we consider, starting from potential $u_{1}$, a binary BT to generate

$$
u_{2}(x)=u_{1}(x)-2 \partial_{x_{1}}^{2} \log \Delta_{2}(x)
$$


or

$$
u_{2}(x)=u_{0}(x)-2 \partial_{x_{1}}^{2} \log \Delta_{1}(x) \Delta_{2}(x)
$$

with

$$
\Delta_{2}(x)=c_{2}+\int_{-\left(\kappa_{2}-\alpha_{2}\right) \infty}^{x_{1}} d x_{1}^{\prime} \phi_{1}\left(x_{1}^{\prime}, x_{2}, i \kappa_{2}\right) \psi_{1}\left(x_{1}^{\prime}, x_{2}, i \alpha_{2}\right),
$$

provided it is possible to choose $\kappa_{2}$ and $\alpha_{2}$ such that the integral is convergent. In the general case, when both $C_{1}\left(i \kappa_{2}\right)$ and $D_{1}\left(i \alpha_{2}\right)$ are different from zero, it can be shown from (4.71) and (4.72) that the integral in (4.77) is convergent if and only if $\kappa_{2}$ and $\alpha_{2}$ are such that the intervals $\left(\alpha_{1}, \kappa_{1}\right)$ and $\left(\alpha_{2}, \kappa_{2}\right)$ have non void intersection. When either one or both are equal to zero less stringent conditions are required. Let us observe that, due to 3.58$)-(3.59)$ and 3.65$)-(3.66$, we have

$$
\begin{aligned}
\lim _{x_{1} \rightarrow-\left(\kappa_{2}-\alpha_{2}\right) \infty} \Delta_{2}(x) & =c_{2} \\
\lim _{x_{1} \rightarrow\left(\kappa_{2}-\alpha_{2}\right) \infty} \Delta_{2}(x) e^{-\left(\kappa_{2}-\alpha_{2}\right) x_{1}-\left(\kappa_{2}^{2}-\alpha_{2}^{2}\right) x_{2}} & =\frac{A_{1}\left(\operatorname{sign}\left(\kappa_{2}-\alpha_{2}\right), i \kappa_{2}\right) B_{1}\left(\operatorname{sign}\left(\kappa_{2}-\alpha_{2}\right), i \alpha_{2}\right)}{\kappa_{2}-\alpha_{2}}
\end{aligned}
$$

where the coefficient is given by $3.62-3.69$ ), that is

$$
\begin{aligned}
& A_{1}\left(\operatorname{sign}\left(\kappa_{2}-\alpha_{2}\right), i \kappa_{2}\right) B_{1}\left(\operatorname{sign}\left(\kappa_{2}-\alpha_{2}\right), i \alpha_{2}\right)= \\
= & 1+\frac{\left(\kappa_{1}-\alpha_{1}\right)\left(\kappa_{2}-\alpha_{2}\right)}{\left(\alpha_{1}-\kappa_{2}\right)\left(\kappa_{2}-\alpha_{1}\right)} \theta\left(\left(\kappa_{2}-\alpha_{2}\right)\left(\kappa_{1}-\alpha_{1}\right)\right) .
\end{aligned}
$$

When $\kappa_{2}$ and $\alpha_{2}$ are both inside the interval of $\kappa_{1}$ and $\alpha_{1}$ or both outside, the asymptotics (4.78) have the same sign at both infinities iff

$$
\left(\kappa_{2}-\alpha_{2}\right) c_{2} \geq 0
$$

while for one inside and the other one outside the interval $\left(\alpha_{1}, \kappa_{1}\right)$ a necessary condition for regularity becomes

$$
\left(\alpha_{1}-\kappa_{1}\right) c_{2} \geq 0
$$

\subsection{Properties of potential}

The easiest way to study properties of potential $(4.76)$ is to solve the recursion relation and express $\Delta_{1} \Delta_{2}$ in terms of the Jost solutions of the original spectral problem. Inserting (4.71) and (4.72) into (4.77) and integrating by parts using the identity

$$
\frac{\phi_{0}\left(x, i \kappa_{1}\right) \psi_{0}\left(x, i \alpha_{1}\right)}{\Delta_{1}(x)}=-\partial_{x_{1}}\left(\frac{1}{\Delta_{1}(x)}\right)
$$

and the following condition

$$
\left(\kappa_{2}-\alpha_{2}\right)\left(\kappa_{1}-\alpha_{1}\right)<0
$$

we can write $\Delta_{1} \Delta_{2}$ as

$$
\Delta_{1}(x) \Delta_{2}(x)=\operatorname{det} A_{2}(x)
$$

where we introduced the $2 \times 2$ matrices $A_{2}, B_{2}$, and $C_{2}$ given by

$$
A_{2}(x)=B_{2}(x)+C_{2}
$$

and $B_{2}$ and $C_{2}$ with entries

$$
c_{11}=c_{1}, \quad c_{22}=c_{2}, \quad c_{12}=C_{1}\left(i \kappa_{2}\right), \quad c_{21}=D_{1}\left(i \alpha_{2}\right)
$$




$$
\left(B_{2}\right)_{j l}(x)=\int_{-\left(\kappa_{l}-\alpha_{j}\right) \infty}^{x_{1}} d x_{1}^{\prime} \phi_{0}\left(x_{1}^{\prime}, x_{2}, i \kappa_{l}\right) \psi_{0}\left(x_{1}^{\prime}, x_{2}, i \alpha_{j}\right) .
$$

Consequently, from (4.76) and (4.83) we obtain for potential $u_{2}$ the following expression

$$
u_{2}=u_{0}-2 \partial_{x_{1}}^{2} \log \operatorname{det} A_{2}(x) .
$$

Due to the result of Prop. 2, it follows from (4.83) that when $\kappa_{2}$ and $\alpha_{2}$ obey (4.82) and are such that the intervals $\kappa_{2}, \alpha_{2}$ and $\kappa_{1}, \alpha_{1}$ are contained one into another, the following conditions

$$
c_{i j}\left(\kappa_{j}-\alpha_{i}\right) \geq 0, \quad i, j=1,2,
$$

ensure that $\Delta_{1} \Delta_{2}$ has no zeros in the $x$-plane and, consequently, that the dressed potential (4.87) is regular. When the two intervals, though having non void intersection, are not contained one into another, these conditions are not sufficient to conclude as before that $\Delta_{1} \Delta_{2}$ has no zeros. Then we'll choose the parameters ordered either according to

$$
\begin{aligned}
& \kappa_{1}<\alpha_{2}<\kappa_{2}<\alpha_{1} \\
& \alpha_{1}<\kappa_{2}<\alpha_{2}<\kappa_{1}
\end{aligned}
$$

or to

$$
\begin{gathered}
\kappa_{2}<\alpha_{1}<\kappa_{1}<\alpha_{2} \\
\alpha_{2}<\kappa_{1}<\alpha_{1}<\kappa_{2} .
\end{gathered}
$$

Now let us compute asymptotic behavior of potential $u_{2}$ along a generic direction $d=$ $x_{1}+h x_{2}$. From (4.83) and using asymptotics of Jost solutions we get that for $x_{2} \rightarrow \pm \infty$ at fixed $d$

$$
\begin{aligned}
& \left(\Delta_{1} \Delta_{2}\right)\left(d-h x_{2}, x_{2}\right) \sim \operatorname{det} C_{2}+ \\
& +\frac{c_{22}}{\kappa_{1}-\alpha_{1}} e^{\left(\kappa_{1}-\alpha_{1}\right) d+\left(\kappa_{1}-\alpha_{1}\right)\left(\kappa_{1}+\alpha_{1}-h\right) x_{2}}+\frac{c_{11}}{\kappa_{2}-\alpha_{2}} e^{\left(\kappa_{2}-\alpha_{2}\right) d+\left(\kappa_{2}-\alpha_{2}\right)\left(\kappa_{2}+\alpha_{2}-h\right) x_{2}} \\
& +\frac{c_{12}}{\alpha_{2}-\kappa_{1}} e^{\left(\kappa_{1}-\alpha_{2}\right) d+\left(\kappa_{1}-\alpha_{2}\right)\left(\kappa_{1}+\alpha_{2}-h\right) x_{2}}+\frac{c_{21}}{\alpha_{1}-\kappa_{2}} e^{\left(\kappa_{2}-\alpha_{1}\right) d+\left(\kappa_{2}-\alpha_{1}\right)\left(\kappa_{2}+\alpha_{1}-h\right) x_{2}} \\
& +K e^{\left(\kappa_{1}-\alpha_{1}+\kappa_{2}-\alpha_{2}\right) d+\left(\kappa_{1}-\alpha_{1}\right)\left(\kappa_{1}+\alpha_{1}-h\right) x_{2}+\left(\kappa_{2}-\alpha_{2}\right)\left(\kappa_{2}+\alpha_{2}-h\right) x_{2}}
\end{aligned}
$$

where we introduced

$$
K=-\left(\kappa_{2}-\kappa_{1}\right)\left(\alpha_{2}-\alpha_{1}\right) \prod_{l, j=1,2} \frac{1}{\kappa_{j}-\alpha_{l}} .
$$

It is tedious but not difficult to prove that along any direction $d=x_{1}+h x_{2}$ with $h \neq \kappa_{i}+\alpha_{j}, \kappa_{i}+\kappa_{i+1}, \alpha_{i}+\alpha_{i+1}$ for $i, j=1,2$ and where $i+1$ is intended modulo 2 , the contribution of $\Delta_{1} \Delta_{2}$ to the potential $u_{2}$ is exponentially decaying.

However, the asymptotic behavior (4.91) along these "special" directions depends widely on matrix $C_{2}$, in the sense that not only the phase shift but also the number and directions of the solitons emerging from the background are determined by the entries of this matrix. We proved that if $c_{i j} \neq 0$ but $c_{i+1, j}=c_{i, j+1}=c_{i+1, j+1}=0$ the potential is decaying along all directions but the one corresponding to $h=\alpha_{i}+\kappa_{j}$ and along this direction it exhibits asymptotically the expected soliton-like behavior. For $c_{i i} \neq 0$ and $c_{i, i+1}=0$ or $c_{i i}=0$ and $c_{i, i+1} \neq 0 i=1,2$ we have two "shifted" solitons along the directions $h=\alpha_{i}+\kappa_{i} i=1,2$ or, respectively, $h=\alpha_{i}+\kappa_{i+1} i=1,2$. When $c_{i i} \neq 0$, 
$c_{i, i+1} \neq 0$ but $c_{i+1, i+1}=c_{i+1, i}=0$ or $c_{i i} \neq 0, c_{i+1, i} \neq 0$ but $c_{i+1, i+1}=c_{i, i+1}=0$ matrix $C_{2}$ is singular and in these cases we have three "tails" along the directions $h=$ $\alpha_{i}+\kappa_{i}, \alpha_{i}+\kappa_{i+1}, \kappa_{i}+\kappa_{i+1}$ or, respectively, $h=\alpha_{i}+\kappa_{i}, \alpha_{i+1}+\kappa_{i}, \alpha_{i}+\alpha_{i+1}$. When all entries but one, say $c_{i j}$, are different from zero, the soliton directions are given by $h=\kappa_{i}+\kappa_{i+1}, \alpha_{i}+\alpha_{i+1}$ and $h=\alpha_{i}+\kappa_{i}, \alpha_{i+1}+\kappa_{i+1}$ if $i \neq j$ and $h=\alpha_{i+1}+\kappa_{i}, \alpha_{i}+\kappa_{i+1}$ if $i=j$. Finally, when $c_{i j} \neq 0$ for all $i, j=1,2$ the potential exhibits asymptotically two "shifted" solitons along the directions $h=\alpha_{i}+\alpha_{i+1}$ and $h=\kappa_{i}+\kappa_{i+1}$.

\subsection{Darboux transform}

As far as the Darboux transforms of $\phi_{1}$ and $\psi_{1}$ are concerned, we can formally define

$$
\phi_{2}(x, k)=\phi_{1}(x, k)-a_{2}(x)\left[C_{2}(k)+\int_{-\left(k_{\Im}-\alpha_{2}\right) \infty}^{x_{1}} d x_{1}^{\prime} \phi_{1}\left(x_{1}^{\prime}, x_{2}, k\right) \psi_{1}\left(x_{1}^{\prime}, x_{2}, i \alpha_{2}\right)\right]
$$

and

$$
\psi_{2}(x, k)=\psi_{1}(x, k)-b_{2}(x)\left[D_{2}(k)+\int_{\left(k_{\Im}-\kappa_{2}\right) \infty}^{x_{1}} d x_{1}^{\prime} \psi_{1}\left(x_{1}^{\prime}, x_{2}, k\right) \phi_{1}\left(x_{1}^{\prime}, x_{2}, i \kappa_{2}\right)\right]
$$

with

$$
a_{2}(x)=\frac{\phi_{1}\left(x, i \kappa_{2}\right)}{\Delta_{2}(x)}, \quad b_{2}(x)=\frac{\psi_{1}\left(x, i \alpha_{2}\right)}{\Delta_{2}(x)} .
$$

However, due to 3.62 ) and (3.69), the integrals in (4.92)-(4.93) are convergent for arbitrary $k$ only if

$$
\left(\alpha_{2}-\kappa_{1}\right)\left(\alpha_{2}-\alpha_{1}\right)<0 \quad\left(\alpha_{1}-\kappa_{2}\right)\left(\kappa_{1}-\kappa_{2}\right)<0,
$$

and these conditions require the parameters to be ordered according to (4.89). In other words, like in KPI case 114, the recursion procedure for getting the solutions is well defined only for a proper choice of order for parameters, even though we proved that the potential is well defined and regular in more general situations.

Now we have to solve the recursion procedure. If we substitute into (4.92) the expressions (4.71) and (4.72) for $\phi_{1}$ and $\psi_{1}$ and integrate by parts using again (4.81), we find

$$
\phi_{2}(x, k)=\frac{1}{\operatorname{det} A_{2}(x)}\left|\begin{array}{lll}
A_{11}(x) & A_{12}(x) & \beta_{1}(x, k)+C_{1}(k) \\
A_{21}(x) & A_{22}(x) & \beta_{2}(x, k)+C_{2}(k) \\
\phi_{0}\left(x, i \kappa_{1}\right) & \phi_{0}\left(x, i \kappa_{2}\right) & \phi_{0}(x, k)
\end{array}\right|
$$

where matrix $A_{2}$ is given by (4.84) and

$$
\beta_{j}(x, k)=\int_{-\left(k_{\Im}-\alpha_{j}\right) \infty}^{x_{1}} d x_{1}^{\prime} \phi_{0}\left(x_{1}^{\prime}, x_{2}, k\right) \psi_{0}\left(x_{1}^{\prime}, x_{2}, i \alpha_{j}\right) .
$$

Clearly, since $\phi_{2}$ given by (4.96) is a solution of the spectral problem for $u_{2}$ for any $C_{1}(k)$ and $C_{2}(k)$, properties of determinants ensure that also

$$
F_{2}(x, k)=\frac{1}{\operatorname{det} A_{2}(x)}\left|\begin{array}{lll}
A_{11}(x) & A_{12}(x) & \beta_{1}(x, k) \\
A_{21}(x) & A_{22}(x) & \beta_{2}(x, k) \\
\phi_{0}\left(x, i \kappa_{1}\right) & \phi_{0}\left(x, i \kappa_{2}\right) & \phi_{0}(x, k)
\end{array}\right|
$$


and

$$
f_{2}(x, k)=\frac{1}{\operatorname{det} A_{2}(x)}\left|\begin{array}{lll}
A_{11}(x) & A_{12}(x) & C_{1}(k) \\
A_{21}(x) & A_{22}(x) & C_{2}(k) \\
\phi_{0}\left(x, i \kappa_{1}\right) & \phi_{0}\left(x, i \kappa_{2}\right) & 0
\end{array}\right|
$$

are solutions of the same spectral problem and

$$
\phi_{2}(x, k)=F_{2}(x, k)+f_{2}(x, k) .
$$

One can easily obtain the analogous expression for dual solutions, that is

$$
\psi_{2}(x, k)=\frac{1}{\operatorname{det} A_{2}(x)}\left|\begin{array}{lll}
A_{11}(x) & A_{12}(x) & \beta_{1}^{d}(x, k)+D_{1}(k) \\
A_{21}(x) & A_{22}(x) & \beta_{2}^{d}(x, k)+D_{2}(k) \\
\psi_{0}\left(x, i \alpha_{1}\right) & \psi_{0}\left(x, i \alpha_{2}\right) & \psi_{0}(x, k)
\end{array}\right|
$$

and

$$
\Upsilon_{2}(x, k)=\frac{1}{\operatorname{det} A_{2}(x)}\left|\begin{array}{lll}
A_{11}(x) & A_{12}(x) & \beta_{1}^{d}(x, k) \\
A_{21}(x) & A_{22}(x) & \beta_{2}^{d}(x, k) \\
\psi_{0}\left(x, i \alpha_{1}\right) & \psi_{0}\left(x, i \alpha_{2}\right) & \psi_{0}(x, k)
\end{array}\right|
$$

where

$$
\beta_{j}^{d}(x, k)=\int_{\left(k_{\Im}-\kappa_{j}\right) \infty}^{x_{1}} d x_{1}^{\prime} \psi_{0}\left(x_{1}^{\prime}, x_{2}, k\right) \phi_{0}\left(x_{1}^{\prime}, x_{2}, i \kappa_{j}\right) .
$$

For $F_{2}$ and $\Upsilon_{2}$ one has the expressions

$$
\begin{aligned}
& F_{2}(x, k)=F_{1}(x, k)-a_{2}(x) \int_{-\left(k_{\Im}-\alpha_{2}\right) \infty}^{x_{1}} d x_{1}^{\prime} F_{1}\left(x_{1}^{\prime}, x_{2}, k\right) \psi_{1}\left(x_{1}^{\prime}, x_{2}, i \alpha_{2}\right), \\
& \Upsilon_{2}(x, k)=\Upsilon_{1}(x, k)-b_{2}(x) \int_{\left(k_{\Im}-\kappa_{2}\right) \infty}^{x_{1}} d x_{1}^{\prime} \Upsilon_{1}\left(x_{1}^{\prime}, x_{2}, k\right) \phi_{1}\left(x_{1}^{\prime}, x_{2}, i \kappa_{2}\right) .
\end{aligned}
$$

Then, if we compute

$$
A_{2}( \pm, k)=\lim _{x_{1} \rightarrow \pm \infty} e^{i k x_{1}+k^{2} x_{2}} F_{2}(x, k)
$$

we find

$$
A_{2}( \pm, k)=A_{1}( \pm, k)+\frac{\kappa_{2}-\alpha_{2}}{i k+\alpha_{2}} A_{1}( \pm, k) B_{1}\left( \pm, i \alpha_{2}\right) \theta\left( \pm\left(\kappa_{2}-\alpha_{2}\right)\right)
$$

where we used that, due to (4.89),

$$
\lim _{x_{1} \rightarrow \pm \infty} \frac{e^{\left(\kappa_{2}-\alpha_{1}\right) x_{1}}}{\left(1+e^{\left(\kappa_{2}-\alpha_{2}\right) x_{1}}\right)\left(1+e^{\left(\kappa_{1}-\alpha_{1}\right) x_{1}}\right)}=0 .
$$

Taking into account (4.82) and (3.69), we can write the following recursion relation

$$
A_{2}( \pm, k)=A_{1}( \pm, k)\left[1+\frac{\kappa_{2}-\alpha_{2}}{i k+\alpha_{2}} \theta\left( \pm\left(\kappa_{2}-\alpha_{2}\right)\right)\right]
$$

Analogously, from 4.105) one can check that $B_{2}( \pm, k)$ defined as

$$
B_{2}( \pm, k)=\lim _{x_{1} \rightarrow \pm \infty} e^{-i k x_{1}-k^{2} x_{2}} \Upsilon_{2}(x, k)
$$


obeys the following recursive relation

$$
B_{2}( \pm, k)=B_{1}( \pm, k)\left[1+\frac{\alpha_{2}-\kappa_{2}}{i k+\kappa_{2}} \theta\left( \pm\left(\kappa_{2}-\alpha_{2}\right)\right)\right] .
$$

Then we obtain the Jost solution and the dual Jost solution for potential $u_{2}$ as

$$
\begin{aligned}
\Phi_{2}(x, k) & =\frac{F_{2}(x, k)}{A_{2}\left(-k_{\Im}, k\right)} \\
\Psi_{2}(x, k) & =\frac{\Upsilon_{2}(x, k)}{B_{2}\left(k_{\Im}, k\right)},
\end{aligned}
$$

since it is easy to check that they satisfy integral equations (2.23) and (2.24) with $u$ substituted by $u_{2}$.

\section{Acknowledgments}

I am very grateful to M. Boiti, F. Pempinelli and A. K. Pogrebkov for help, suggestions and fruitful discussions.

\section{References}

[1] B. B. Kadomtsev and V. I. Petviashvili, Sov. Phys. Doklady 192, 539 (1970).

[2] V. S. Dryuma, Sov. Phys. J. Exp. Theor. Phys. Lett. 19, 381 (1974).

[3] V. E. Zakharov and A. B. Shabat, Funct. Anal. Appl. 8, 226 (1974).

[4] M. Ablowitz, D. Bar Yacoov, A. S. Fokas, Stud. Appl. Math. 69, 135 (1983).

[5] V. E. Zakharov, S. V. Manakov, Sov. Sci. Rev. Phys. Rev. 1, 133 (1979).

[6] S. V. Manakov, Physica D3, 420 (1981).

[7] A. S. Fokas and M. J. Ablowitz, Stud. Appl. Math. 69, 211 (1983).

[8] P. G. Grinevich, S. P. Novikov, Func. Anal. Appl. 22, 23 (1988).

[9] M. Boiti, F. Pempinelli, A. K. Pogrebkov, and M. C. Polivanov, Theor. Math. Phys. 93, 1200 (1992).

[10] M. Boiti, F. Pempinelli, and A. Pogrebkov, Theor. Math. Phys. 99, 511 (1994).

[11] M. Boiti, F. Pempinelli, and A. Pogrebkov, in Nonlinear Physics. Theory and Experiment, edts. E. Alfinito, M. Boiti, L. Martina, and F. Pempinelli, World Scientific Pub. Co., Singapore (1996), pp. 37-52.

[12] M. Boiti, F. Pempinelli, and A. Pogrebkov, Inverse Problems 13, L7 (1997).

[13] M. Boiti, F. Pempinelli, A. Pogrebkov, and B. Prinari, Theor. Math. Phys. 116, 741 (1998).

[14] M. Boiti, F. Pempinelli, A.K. Pogrebkov, B. Prinari, Proceedings of the Steklov Institute of Mathematics 126, 42-62 (1999). 
[15] M. J. Ablowitz and P. A. Clarkson, Solitons, nonlinear evolution equations and inverse scattering, Lecture Notes Series 49, University of Cambridge, Cambridge (1991).

[16] M.V. Wickerhauser, Commun. Math. Phys. 108, 67-89 (1987).

[17] V. B. Matveev and M. A. Salle, Darboux Transformations and Solitons, Springer, Berlin (1991).

[18] M. A. Salle, PhD Thesis, Leningrad.

[19] M. Boiti, F. Pempinelli, A. K. Pogrebkov, and M. C. Polivanov, Inverse problems 7, 43 (1991). 